\section{A) Check for updates}

Cite this: Food Funct., 2021, 12, 9680

\title{
Effect of roasting conditions on cocoa bioactivity and gut microbiota modulation
}

\author{
Lida Yaneth Maldonado-Mateus, ${ }^{\text {a,b }}$ Sergio Perez-Burillo, a,c,d \\ Alberto Lerma-Aguilera, ${ }^{e}$ Daniel Hinojosa-Nogueira, ${ }^{a}$ Sonia Ruíz-Pérez, (D) e

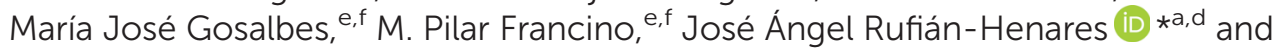 \\ Silvia Pastoriza de la Cueva ${ }^{a}$
}

\begin{abstract}
Cocoa is a highly consumed food with beneficial effects on human health. Cocoa roasting has an important influence on its sensory and nutritional characteristics; therefore, roasting could also play a role in cocoa bioactivity. Thus, the aim of this paper is to unravel the effect of cocoa roasting conditions on its antioxidant capacity and modifications of gut microbiota after in vitro digestion-fermentation. HMF and furfural, chemical markers of non-enzymatic browning, were analyzed in unroasted and roasted cocoa powder at different temperatures, as well as different chocolates. The antioxidant capacity decreased with roasting, most probably due to the loss of phenolic compounds during heating. In the case of the evaluated chocolates, the antioxidant capacity was 2-3 times higher in the fermented fraction. On the other hand, HMF and furfural content increased during roasting due to increasing temperatures. Moreover, unroasted and roasted cocoa powder have different effects on gut microbial communities. Roasted cocoa favored butyrate production, whereas unroasted cocoa favored acetate and propionate production in a significant manner. In addition, unroasted and roasted cocoa produced significantly different gut microbial communities in terms of composition. Although many bacteria were affected, Veillonella and Faecalibacterium were some of the most discriminant ones; whereas the former is a propionate producer, the latter is a butyrate producer that has also been linked to positive effects on the inflammatory health of the gut and the immune system. Therefore, unroasted and roasted cocoa (regardless of the roasting temperature) promote different bacteria and a different SCFA production.
\end{abstract}

Received 15th April 2021, Accepted 2nd August 2021 DOI: $10.1039 / \mathrm{d} 1$ fo0 $1155 \mathrm{c}$ rsc.li/food-function

\section{Introduction}

Cocoa and its related products are heavily consumed worldwide, mostly due to their pleasant taste. In fact, cocoa beans exported in 2017 reached a market value of USD 8.6 billion and this is expected to keep growing, reaching a value of USD 16.3 billion in $2025 .{ }^{1}$ On the other hand, the chocolate industry had a market value of USD 106.19 billion in 2017 and is expected to grow to USD 189.9 billion by $2026 .{ }^{1}$ These figures

\footnotetext{
${ }^{a}$ Departamento de Nutrición y Bromatología, Instituto de Nutrición y Tecnología de los Alimentos, Centro de Investigación Biomédica, Universidad de Granada, Granada, Spain.E-mail: jarufian@ugr.es; Tel: +34 9582428 41;

Fax: +34958249577

${ }^{b}$ Grupo GIBA, Universidad de Pamplona, Colombia

${ }^{c}$ Department of Biochemistry and Molecular Biology, Boonshoft School of Medicine, Wright State University, Dayton, OH, USA

${ }^{d}$ Instituto de Investigación Biosanitaria ibs.GRANADA, Universidad de Granada, Granada, Spain

${ }^{e}$ Área de Genómica y Salud, Fundación para el Fomento de la Investigación Sanitaria y Biomédica de la Comunitat Valenciana-Salud Pública, Valencia, Spain

${ }^{f}$ CIBEResp, Madrid, Spain
}

just give a sense of the importance of the cocoa and chocolate industry around the world.

Considering this information, cocoa and chocolate have not gone unnoticed by the scientific community and many studies have been carried out to investigate possible benefits on human health. As such, cocoa has demonstrated anti-inflammatory activity, positive effects on the immune system or on cancer treatment, among others. ${ }^{2}$ Many of these benefits have been linked to the polyphenolic content of cocoa, which has been extensively studied and is characterized by high concentrations of flavan-3-ols (catechin and epicatechin) and procyanidins B1 and B2 and other compounds in lower amounts. ${ }^{3}$ Moreover, not only have naturally present phenolic compounds been proven to be beneficial, but also their gut microbial metabolites, especially different isomers of dihydroxyphenyl-gamma-valerolactone, have demonstrated biological activities, ${ }^{4,5}$ as well as methylxanthines, ${ }^{6}$ and fibers. However, the different processing steps that occur between harvest and the final cocoa powder, including fermentation, drying, alkalization and roasting, all have a significant impact on cocoa powder final composition. ${ }^{7}$ Whereas fermentation 
and roasting are essential for the development of the final flavor and color, ${ }^{8,9}$ both processes will cause a loss in polyphenols (catechins, epicatechins and procyanidins) due to oxidation, polymerization and degradation. ${ }^{10-12}$ However, some of them will still remain intact while some others will bind with proteins to form tannins, responsible for cocoa astringency. These polymers can also be substrates for gut microbes. ${ }^{13}$ Additionally, during roasting non-enzymatic browning gives rise to different chemical reactions ${ }^{14,15}$ and the generation of high-molecular weight compounds named melanoidins, which exert a potent antioxidant activity. ${ }^{16}$ Such melanoidins also reach the colon, where they can be degraded by beneficial bacteria promoting their growth and metabolism. ${ }^{17,18}$

Accordingly, cocoa is a source of different compounds that can be used by gut microbes and therefore potentially modulate their growth and metabolic activity. As such, the phenolic metabolism by gut microbes has been extensively studied both in vitro and in vivo. ${ }^{3,5,19}$ While most research studies involving cocoa and gut microbiota are focused on either a particular bioactive compound or a cocoa formula enriched in some phytochemicals, the effect that cocoa powder itself could have on gut microbiota has barely been studied. Massot-Cladera ${ }^{20}$ studied the effect of a cocoa-rich diet in rats but gut microbiota was examined via FISH (fluorescence in situ hybridization) and therefore, only targeted bacteria were accounted for. On the other hand, Álvarez-Cilleros et al. ${ }^{21}$ investigated how a cocoa-rich diet affected the gut microbiota of diabetic rats.

Therefore, since cocoa roasting results in a loss of bioactive compounds, the aim of this research is to investigate whether unroasted cocoa powder has a different bioactivity (i.e. antioxidant capacity and effect on gut microbial communities) from roasted cocoa powder. The roasting temperature is also studied to establish, if any, the best temperature to generate a stronger antioxidant capacity and a healthier gut microbiota profile. All these assays were performed after in vitro digestion and gut microbial fermentation to parallel the modifications occurring along the human gastrointestinal tract.

\section{Materials and methods}

\subsection{Samples}

Cocoa samples belonged to the "forastero" variety (Theobroma cacao spp. sphaerocarpum) harvested in 2019 from two different locations, Tibú and Zulia, Colombia. For each sample, $1 \mathrm{~kg}$ of cocoa was harvested at random. Cocoa beans were afterwards classified to remove any damaged beans or those with flaws or defects. Cocoa beans were processed to obtain the cocoa powder, which was then frozen and shipped to the University of Granada (Spain). Cocoa beans processing included a roasting step carried out at different temperatures for 30 minutes: $50,70,90$ and $120{ }^{\circ} \mathrm{C}$. However, we have to state that according to Beg et al. ${ }^{8}$ cocoa is industrially roasted at a temperature between 90 and $145{ }^{\circ} \mathrm{C}$. Accordingly, cocoa samples included 4 ( 2 for each location) unroasted or raw cocoa samples and 4 roasted samples for each roasting temperature (all samples were analyzed in duplicate).

Nine different chocolates (8 dark and 1 mixed with milk) were manufactured and processed by the company Somos Cacao SAS (Colombia). The content of cocoa solids or cocoa content, according to the label, was in the range of 40 to $80 \%$. All samples were stored at $-80^{\circ} \mathrm{C}$ and shipped in dry ice to the University of Granada for subsequent analyses.

\subsection{Reagents}

Trolox (( \pm$)-6$-hydroxy-2,5,7,8-tetramethylchromane-2-carboxylic acid), 2,4,6-tri(2-pyridyl)-s-triazine (TPTZ), gallic acid, acetic acid, propionic acid, butyric acid, Folin-Ciocalteu reagent, sodium hydroxide, hydrochloric acid, ferric chloride hexahydrate, sodium acetate, potassium chloride, potassium dihydrogen phosphate, sodium monohydrogen carbonate, sodium chloride, magnesium chloride hexahydrate, ammonium carbonate, calcium chloride dihydrate, sodium dihydrogen phosphate, tryptone, cysteine, sodium sulphide, resazurin, salivary $\alpha$-amylase, porcine pepsin, bile acids (porcine bile extract), pancreatin and ethanol were from Sigma-Aldrich (Darmstadt, Germany).

\subsection{In vitro gastrointestinal digestion and in vitro gut microbial fermentation}

2.3.1. In vitro gastrointestinal digestion. In vitro gastrointestinal digestion was carried out according to the protocol described by Brodkorb et al. ${ }^{22}$ Five grams of cocoa powder was mixed $\left(1: 1 \mathrm{w} \mathrm{vol}^{-1}\right)$ with $5 \mathrm{~mL}$ of simulated salivary fluid containing $150 \mathrm{U} \mathrm{mL}^{-1}$ of $\alpha$-amylase and kept at $37{ }^{\circ} \mathrm{C}$ in oscillation for 2 minutes. Then, $10 \mathrm{~mL}$ of simulated gastric fluid with $4000 \mathrm{U} \mathrm{mL}^{-1}$ of pepsin was added and the $\mathrm{pH}$ was adjusted to 3 . The mixture was kept at $37^{\circ} \mathrm{C}$ in oscillation for 2 hours. Finally, $20 \mathrm{~mL}$ of simulated intestinal fluid with 200 $\mathrm{U} \mathrm{mL}^{-1}$ of pancreatin and bile salts of concentration $20 \mathrm{mM}$ were added and the $\mathrm{pH}$ was adjusted to 7 . The mixture was kept at $37{ }^{\circ} \mathrm{C}$ in oscillation for 2 hours. Digestion was halted by immersion of the tubes in ice for 15 minutes. The tubes were afterwards centrifuged at $3500 \mathrm{~g}$ for 10 minutes and aliquots of the supernatant were taken and stored at $-80^{\circ} \mathrm{C}$ until further analysis. The solid pellet or solid residue, representing the fraction that reaches the large intestine, was used as input for in vitro gut microbial fermentation.

2.3.2. In vitro gut microbial fermentation. In vitro gut microbial fermentation was carried out according to the protocol described by Pérez-Burillo et $a l^{23,24}$ Briefly, fecal material was collected the morning of the experiment from 4 healthy volunteers. They had an average BMI (body mass index) of 22.6, they had no history of intestinal disease, they had not taken antibiotics for at least the 6 previous months and they were not taking any pro- or prebiotics. Fecal materials from all 4 volunteers were pooled together to minimize inter-individual variability. The fecal inoculum was obtained by adding $0.1 \mathrm{M}$ phosphate buffer at pH 7 to the fecal material to obtain a $32 \%$ $\left(\mathrm{w} \mathrm{vol}^{-1}\right)$ inoculum. The fermentation medium was composed of peptone $\left(14 \mathrm{~g} \mathrm{~L}^{-1}\right)$ at $\mathrm{pH} 7,0.312 \mathrm{~g} \mathrm{~L}^{-1}$ of cysteine and 
$0.312 \mathrm{~g} \mathrm{~L}^{-1}$ of hydrogen sulfide. $0.5 \mathrm{~g}$ of undigested residue were weighted into tubes and $7.5 \mathrm{~mL}$ of fermentation medium was added. Finally, $2 \mathrm{~mL}$ of fecal inoculum was added to each tube. The whole process was carried out inside an anaerobic chamber keeping $\mathrm{N}_{2}, \mathrm{CO}_{2}$, and $\mathrm{H}_{2}$ gas concentrations at $80 \%$, $16 \%$ and $4 \%$. The tubes were incubated at $37{ }^{\circ} \mathrm{C}$ for 20 hours in oscillation. The fermentation was halted by immersion in ice. The tubes were afterwards centrifuged at $3500 \mathrm{~g}$ for 10 minutes and aliquots of the supernatant (potentially absorbable fraction) were taken and stored at $-80^{\circ} \mathrm{C}$ until further analysis. In vitro fermentation was carried out in duplicate.

\subsection{Antioxidant capacity assays}

2.4.1. FRAP assay. The reducing capacity of the digested and fermented samples was measured according to the procedure described by Benzie \& Strain, ${ }^{25}$ and adapted to a microplate reader (FLUOStar Omega, BMG Labtech, Germany). Briefly, each well of a 96-well plastic plate contained $280 \mu \mathrm{L}$ of FRAP reagent and $20 \mu \mathrm{L}$ of the sample (both supernatants were obtained after in vitro digestion and in vitro fermentation). The FRAP reagent was composed of $25 \mathrm{~mL}$ of $0.3 \mathrm{mM}$ sodium acetate buffer at pH 3.6, $2.5 \mathrm{~mL}$ of a $10 \mathrm{mM}$ solution of 2,4,6tri(2-pyridyl)-s-triazine (TPTZ) dissolved in $\mathrm{HCl} 40 \mathrm{mM}$ and $2.5 \mathrm{~mL}$ of $20 \mathrm{mM}$ ferric chloride solution. The absorbance readings were taken every 60 seconds for 30 minutes at $595 \mathrm{~nm}$. A calibration curve with Trolox was prepared in the range of $0.01-0.4 \mathrm{mg} \mathrm{mL^{-1 }}$. The results were expressed as mmol Trolox equivalents $\mathrm{kg}^{-1}$ of the sample.

2.4.2. DPPH assay. The antiradical activity of the digested and fermented samples was measured according to the procedure described in Navajas-Porras et $a .^{26}{ }^{26}$ Briefly, $280 \mu \mathrm{L}$ of DPPH solution $\left(7.4 \mathrm{mg} 100 \mathrm{~mL}^{-1}\right.$ of methanol) was mixed with $20 \mu \mathrm{L}$ of the sample (both supernatants obtained after in vitro digestion and in vitro fermentation) in a 96-well plastic plate. Absorbance readings were taken every 60 seconds for 60 minutes at $515 \mathrm{~nm}$. A FLUOStar Omega microplate reader was used (BMG Labtech, Germany). A calibration curve with Trolox was prepared in the range of $0.01-0.4 \mathrm{mg} \mathrm{mL}^{-1}$. The results were expressed as mmol Trolox equivalents/kg of the sample.

2.4.3. Folin-Ciocalteu assay. Folin-Ciocalteu assay was carried out as described by Singleton \& Rossi, ${ }^{27}$ but adapted to a microplate reader (FLUOStar Omega, BMG Labtech, Germany). Briefly, in a 96-well plastic plate, $15 \mu \mathrm{L}$ of FolinCiocalteu reagent was mixed with $60 \mu \mathrm{L}$ of sodium carbonate (10\%), $195 \mu \mathrm{L}$ of Milli-Q water and $30 \mu \mathrm{L}$ of the sample (both supernatants obtained after in vitro digestion and in vitro fermentation). The absorbance readings were taken every 60 seconds for 60 minutes at $765 \mathrm{~nm}$. A calibration curve with gallic acid was prepared in the range of $10-100 \mathrm{mg} \mathrm{L}^{-1}$. The results were expressed as $\mathrm{mg}$ gallic acid equivalents per $\mathrm{kg}$ of the sample.

\subsection{5 -Hydroxymethylfurfural and furfural analyses}

HMF analysis was carried out as described by Rufián-Henares et $a .^{28}$ Cocoa powder and chocolate samples were suspended in Milli-Q water and clarified with Carrez I (potassium ferrocyanide, $15 \% \mathrm{w} \mathrm{v}^{-1}$ ) and Carrez II (zinc acetate $30 \% \mathrm{w} \mathrm{v}^{-1}$ ) solutions. The resulting supernatant was filtered through a $0.22 \mu \mathrm{m}$ nylon filter. The filtrate was injected into an HPLC system (Accela 600). The HPLC system was composed of a PDA detector, a multisampler and a quaternary pump. An RP $\mathrm{C}_{18}$ column was used. As mobile phase, $5 \%$ acetonitrile in water was used. The flow rate was $1 \mathrm{~mL} \mathrm{~min}^{-1}$ and the wavelength was $284 \mathrm{~nm}$. The results were expressed as $\mathrm{mg} \mathrm{kg}^{-1}$ of sample.

\subsection{Short chain fatty acid analysis}

Short chain fatty acids were analyzed as in Pérez-Burillo et al. ${ }^{18}$ In vitro fermentation supernatant was filtered through a $0.22 \mu \mathrm{m}$ nylon filter and injected into the HPLC system (Accela 600). The HPLC system was composed of a PDA detector, a multisampler and a quaternary pump. The detector was set at $210 \mathrm{~nm}$; the mobile phase used was Milli-Q water with 1\% of formic acid/acetonitrile with $1 \%$ of formic acid at a rate of $99: 1 \mathrm{v} \mathrm{v}^{-1}$ delivered at a $1.25 \mathrm{~mL} \mathrm{~min}^{-1}$ flow rate; the column used was an Aquasil $\mathrm{C}_{18}$ reverse phase (Thermo Scientific) (150 $\times 4.6 \mathrm{~mm}, 5 \mu \mathrm{m}$ ), with a total run time of $30 \mathrm{~min}$. Calibration curves with standard acetate, propionate and butyrate were made at a concentration range of $10-0.01 \mathrm{mM}$. The results were expressed in $\mathrm{mM}$.

\subsection{S rRNA amplicon sequencing and bioinformatic analysis}

DNA extraction from the solid residues derived from the fermentation process was performed using a MagNA Pure LC JE379 platform (Roche) and DNA Isolation Kit III, with initial lysis using lysozyme at $0.1 \mathrm{mg} \mathrm{ml} \mathrm{ml}^{-1} .12 \mathrm{ng}$ of microbial genomic DNA was used as the template for the amplification of the V3-V4 hypervariable region of the 16S rRNA gene, following the Illumina protocol for $16 \mathrm{~S}$ Metagenomic Sequencing Library Preparation (Cod. 15044223 Rev. A). The PCR primers used were as described by Klindworth et al., ${ }^{29}$ with the forward primer being 5'-TCGT CGGC AGCG TCAG ATGT GTAT AAGA GACA GCCT ACGG GNGG CWGCA-G3' and the reverse primer being 5'-GTCT CGTG GGCT CGGA GATG TGTA TAAG AGAC AGGA CTAC HVGG GTAT CTAA TCC3'. The primers contained adapter sequences to make them compatible with the Illumina Nextera XT Index kit. Amplicon libraries were pooled and sequenced in an Illumina MiSeq sequencer in a $2 \times 300$ cycles paired-end run (MiSeq Reagent kit v3).

The DADA2 (v1.8.0) package ${ }^{30}$ from R (v3.6.0) was employed for sequence processing and read joining, as well as for the generation and annotation of amplicon sequence variants (ASVs). The filtering and trimming parameters were as follows: $\operatorname{maxN}=0, \operatorname{maxEE}=c(2,5)$, truncQ $=0$, trimLeft $=c(17,21)$, truncLen $=c(270220)$, and rm.phix $=$ TRUE. A minimum overlap of 15 nucleotides and a maximum mismatch of 1 were required for the merging of forward and reverse sequencing reads. Bowtie $2^{31}$ was employed to align the reads against the human genome (GRCh38.p11) and the matches were discarded. Sequences with $100 \%$ similarity were clustered into 
ASVs that were taxonomically annotated through comparison to the SILVA132 reference database (v1.12). ${ }^{32}$

\subsection{Statistics}

Principal Coordinates Analysis (PCoA) with the Bray-Curtis dissimilarity index was carried out for the exploratory analysis of 16S rRNA sequencing data. Canonical Correspondence Analysis (CCA) and coinertia analysis were applied as interpretative methods. Both methodologies try to find associations between the two sets of variables; in the case of CCA, it tries to explain the variability found in the microbial community via several environmental explanatory variables. In the case of coinertia analysis, it tries to find associations between the microbial community and SCFA; in this case, by finding the correlation between the metabolite PCA and the microbial PCoA. The strength of the association found with coinertia analysis is measured via the RV coefficient. It is a number between 0 and 1 and the higher the coefficient the stronger the association. ${ }^{33}$ Random Forest was used as discriminant analysis. The importance that the random forest algorithm gives to each feature (i.e.: each genus) to classify samples, in this case, as roasted or unroasted, is given by two parameters: mean decrease accuracy and mean decrease Gini. Mean decrease accuracy gives a rough estimate of the loss in prediction performance when that particular variable is omitted from the training set. Mean decrease
Gini is a measure of node impurity; a node with the highest purity is that which has only features of the same class (roasted vs unroasted). By calculating the decrease in Gini when a feature is omitted, we learn the importance of such feature for classification of the data as roasted or unroasted. ${ }^{34}$ Statistical significance was set at $p<0.05$. Significance between pairs of categories in antioxidant capacity, metabolites and genus abundance was tested by the Wilcoxon test. All analyses were carried out in $\mathrm{R}$ version 3.6.3.

\section{Results and discussion}

\subsection{Antioxidant capacity of raw and roasted cocoa}

Cocoa samples from two different locations (raw and roasted at different temperatures) were subjected to in vitro gastrointestinal digestion followed by in vitro fermentation. We obtained two supernatants: one resembling the potentially absorbable fraction in the small intestine and another resembling the fraction that can be absorbed in the large intestine. Antioxidant capacity was evaluated in both fractions by means of DPPH, FRAP and Folin-Ciocalteu methods.

The antioxidant capacity released after in vitro gut microbial fermentation (Fig. 1) was always higher than that released after in vitro digestion, though the difference was significant $(p<$

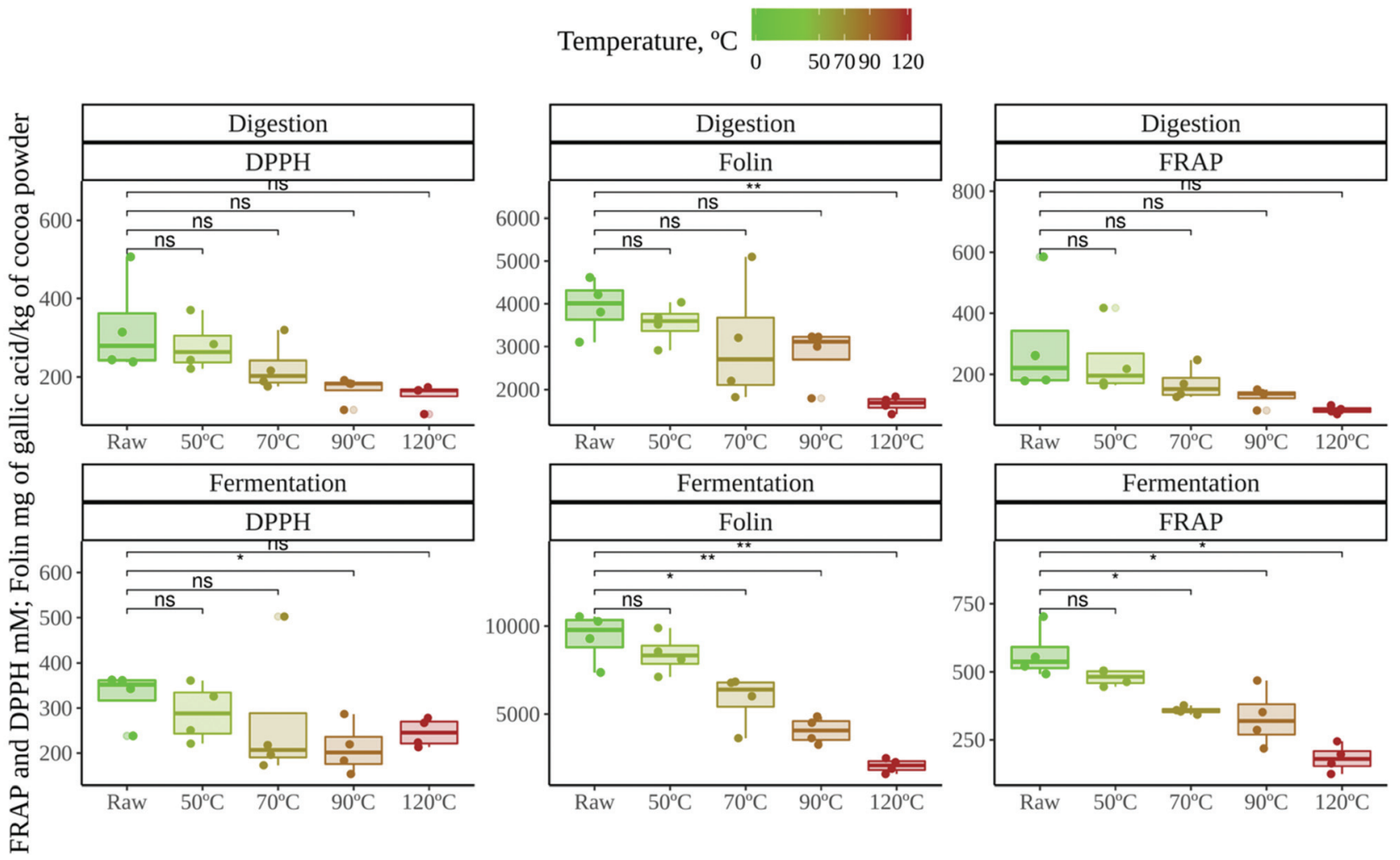

Fig. 1 Antioxidant capacity of raw (unroasted) and roasted cocoa powders at different temperatures. The upper three panels show the antioxidant capacity obtained from the in vitro digestion supernatant. The bottom three panels show the antioxidant capacity obtained from the in vitro gut microbial fermentation supernatant. Significance was tested via the Wilcoxon test $(p<0.05)$. Comparisons were made using raw cocoa as the reference group. Significance labels: ns: not significant; *: $p<0.05 ; * *: p<0.01$. 
0.05) only in the case of the FRAP and Folin-Ciocalteu methods (statistical differences not shown in Fig. 1). This has been previously reported, especially in the case of plant derived foods, where bioactive components are less accessible to human digestive enzymes. ${ }^{26,35}$ In addition, cocoa has an impressive load of phenolic compounds, mostly flavan-3-ols and procyanidins, which reach the colon and are extensively metabolized by gut microbes into smaller molecules. ${ }^{36}$ Therefore, they could increase the antioxidant capacity of this fraction.

The antioxidant capacity, on the other hand, was no different depending on the location of the cocoa crop (data not shown). However, roasting did have a strong influence on antioxidant capacity, regardless of the assay. Raw cocoa samples were significantly more antioxidant than roasted cocoa samples. In fact, as depicted in Fig. 1, the antioxidant capacity decreased as the roasting temperature increased. This has been previously described in undigested-unfermented cocoa samples, ${ }^{37,38}$ and it has been linked to the loss of phenolic compounds that occurs during roasting. ${ }^{6}$ In our study, this loss of antioxidant power was more apparent in the fermentation supernatant. A potential explanation could be that the antioxidant capacity released during gut microbial fermentation was highly dependent on those metabolites derived from flavan-3-ols and procyanidins, which could reach the colon in much lower concentrations due to roasting.

\subsection{HMF and furfural as thermal damage markers of roasted} cocoa

HMF and furfural are chemical compounds produced during non-enzymatic browning (Maillard reaction) and caramelization. ${ }^{39}$ Therefore, they are often used as quality parameters to control roasting or heating processes in foods, ${ }^{39,40}$ also in cocoa. ${ }^{41}$ Our results showed that the location of the crop did not have any influence on the formation of HMF or furfural during roasting (data not shown). This is not surprising since their formation will depend on common ingredients (reducing sugars, amino acids or vitamins) whose concentrations should be barely affected by the crop location.

On the other hand, HMF and furfural concentrations increased during roasting (Fig. 2), which is in agreement with other studies. ${ }^{41}$ In the case of furfural, the largest production was observed till $70{ }^{\circ} \mathrm{C}$ and then a decrease of furfural generation was observed. This could be explained by taking into account that furfural is a volatile compound, so that it can be volatilized with a hardest roasting process (contrary to HMF, which is not volatile). HMF has proven, in animals, to be hepato- and nephrotoxic via previous formation of 5-sulfooxymethylfurfural (SMF). ${ }^{42}$ Therefore, these chemical compounds could be useful in controlling the roasting process, not only of cocoa but other foods too, with
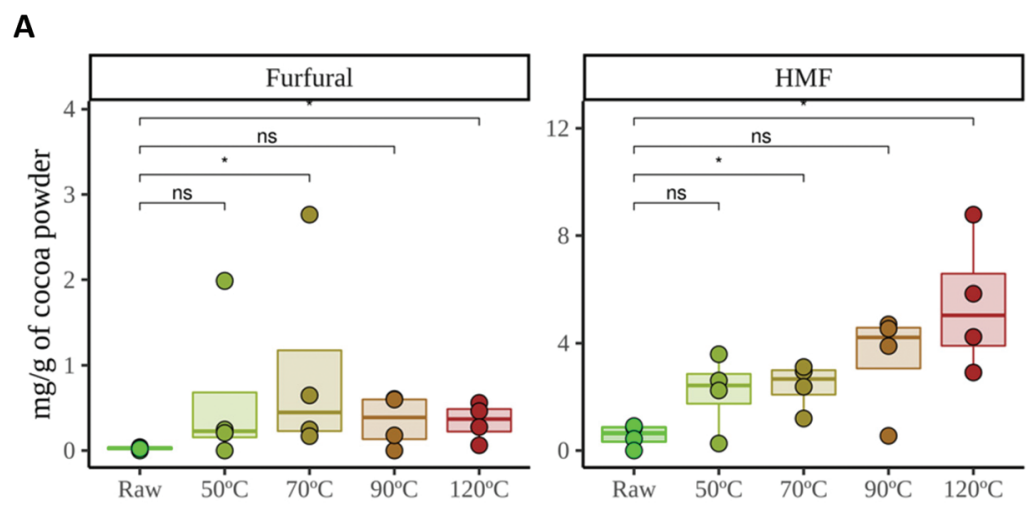

B
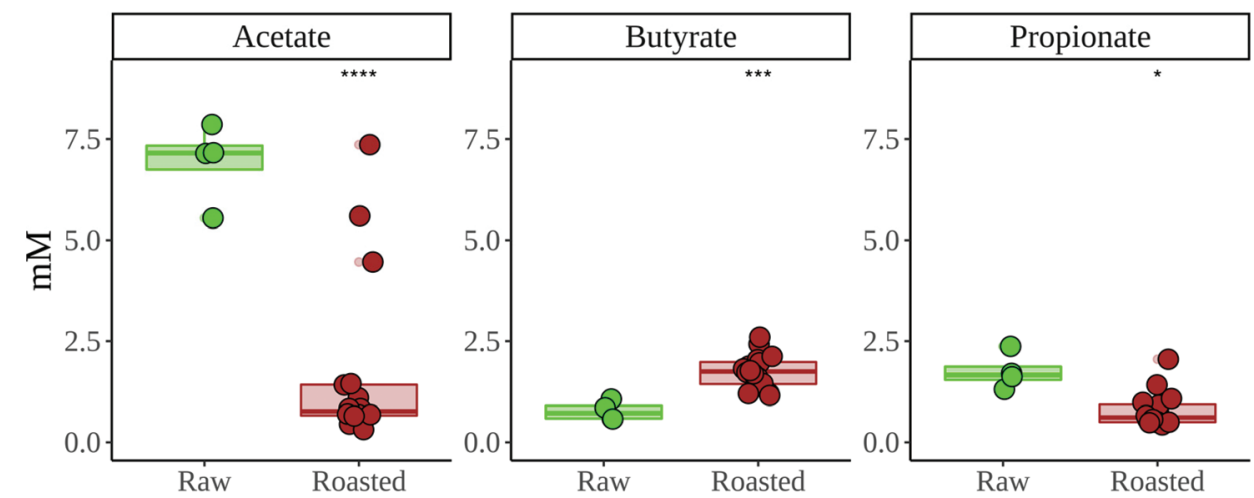

Fig. 2 (A) HMF and furfural concentrations of raw and roasted cocoa powders. (B) Short chain fatty acid concentration after in vitro gut microbial fermentation of raw and roasted cocoa powders. Significance was tested via the Wilcoxon test $(p<0.05)$. Comparisons were made using raw cocoa as the reference group. Significance labels: ns: not significant; *: $p<0.05 ;{ }^{* *}: p<0.01$. 
the aim to reduce HMF content and its potential health damaging effect.

\subsection{Gut microbiota community structure and functionality}

The gut microbial communities resulting from in vitro fermentation of raw and roasted cocoa powder obtained from two different locations were also studied. Gut microbiota community structure or composition (i.e. the abundance of each bacterial genus) was investigated by $16 \mathrm{~S}$ rRNA gene sequencing whereas their functionality was assessed by analyzing short chain fatty acid (SCFA) production since they are known to be the main gut microbial fermentation products. ${ }^{43}$
As shown by PCoA with Bray-Curtis dissimilarity (Fig. 3A), gut microbial communities seemed to be especially affected by roasting, with a large dissimilarity between raw and roasted (regardless of the temperature) cocoa samples. The location of the crop, however, did not seem to play a differential role in the structure of the microbial community, though some dissimilarity between locations can be noticed in Fig. 3A. Then a canonical correspondence analysis (CCA) was performed using the following as the explanatory variables of the gut microbial community structure: (i) location of the crop; (ii) whether cocoa was roasted or raw; and (iii) HMF and furfural concentrations (Fig. 3B). This interpretative analysis is usually applied
A Temperature, ${ }^{\circ} \mathrm{C} \underset{0}{1507090120}$ Location $\bigcirc$ Zulia $\triangle$ Tibu

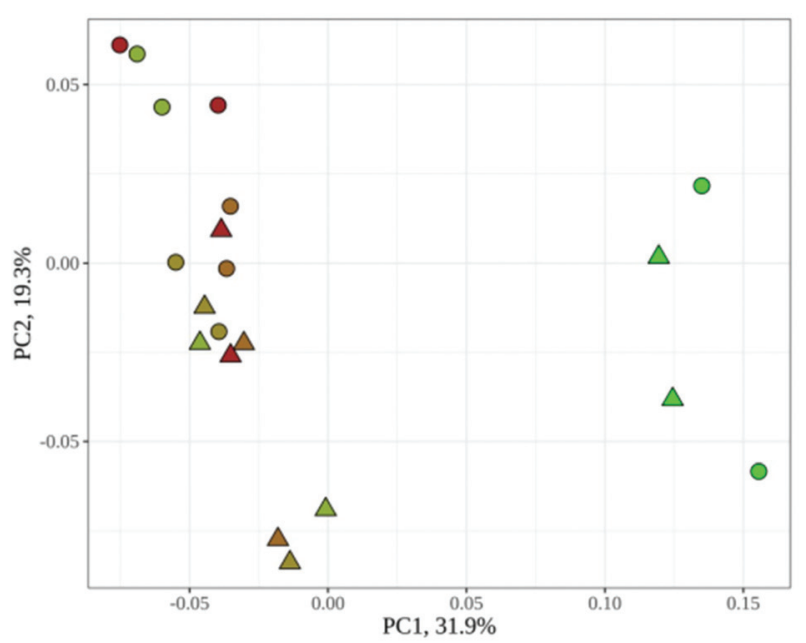
Temperature, ${ }^{\circ} \mathrm{C} \underset{0}{1} 507090 \quad 120$

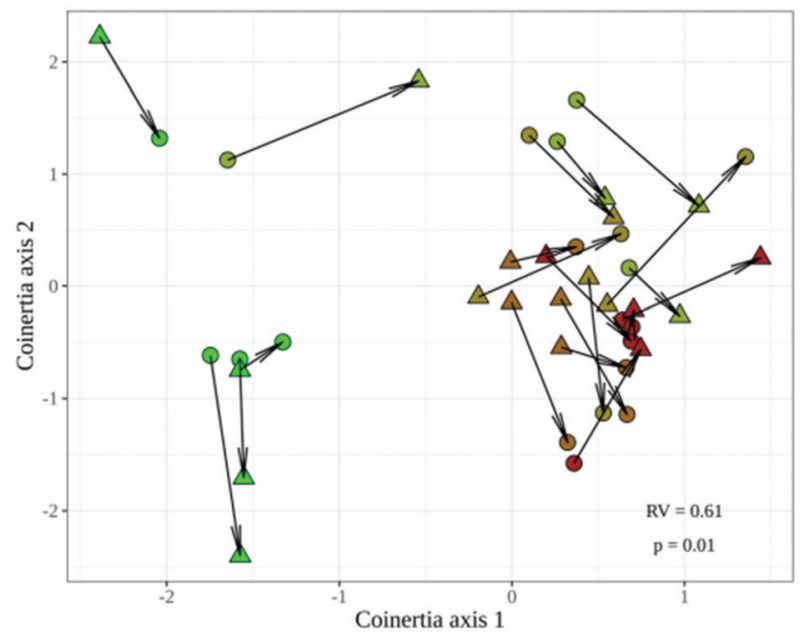

\section{C} $\bigcirc$ SCFA $\triangle$ Species
B
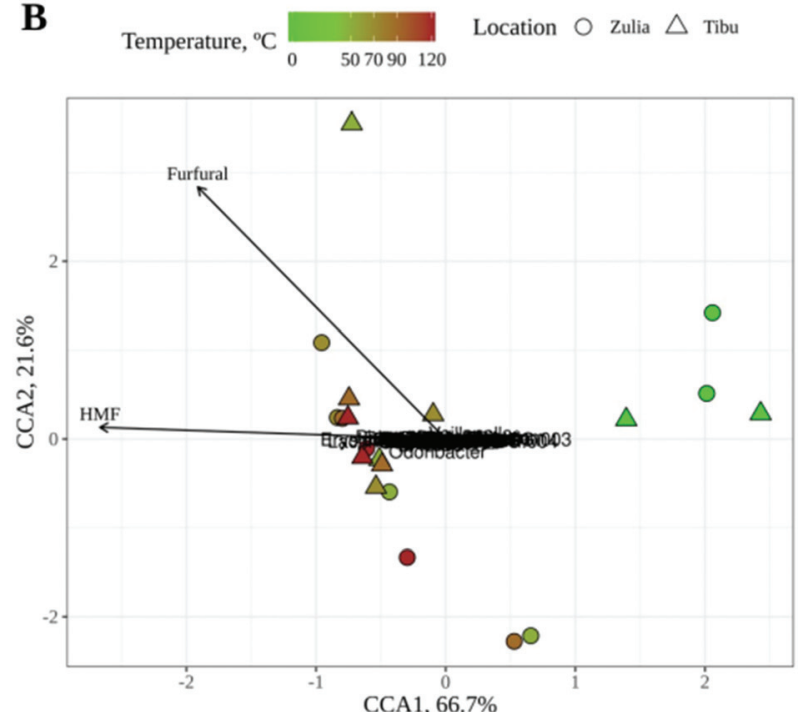

Fig. 3 (A) Principal Coordinates Analysis (PCoA) with Bray-Curtis dissimilarity of the gut microbial community structure after in vitro gut microbial fermentation of raw and roasted cocoa powders. (B) Canonical Correspondence Analysis (CCA) of the gut microbial community structure after in vitro gut microbial fermentation of raw and roasted cocoa powder using as explanatory variables raw/roasted, location of the crop, and $\mathrm{HMF}$ and furfural concentrations. The arrows point in the direction at which metabolite concentrations are higher. (C) Coinertia analysis between the SCFAbased PCA and the genus-abundance-based PCoA. The significancy of the model is given by a $p$ value $<0.01$ and a RV = 0.61 (level of correlation). 
to find associations between explanatory variables and the response variables (in this case, gut microbial community composition). According to CCA, whether cocoa samples were roasted or not had a significant influence $(p=0.001)$ on the composition of the microbial community whereas location $(p$ $=0.330)$ and HMF $(p=0.407)$ and furfural $(p=0.063)$ concentrations did not. It makes sense that roasting could have a significant influence on shaping gut microbial communities. During this process, the cocoa chemical composition is dramatically changed and so could be the substrates that reach the colon. On one hand, during roasting, the phenolic profile changes as previously described. ${ }^{6}$ Moreover, and probably more importantly, during roasting there will be a formation of melanoidins as end products of the chemical browning, which are not present in raw cocoa. These polymers have proven to behave as fibers (including cocoa melanoidins), reaching the colon where they can be degraded by gut bacteria. ${ }^{17,18}$ In addition, it has been described how the roasting process of coffee beans translates into a loss of poly- and oligosaccharides. $^{44}$ Cocoa bean poly- and oligosaccharides could likely suffer the same fate during roasting. Therefore, given that fiber and other polysaccharides are the main gut bacterial substrate, ${ }^{45}$ it is not surprising that roasting was found to profoundly impact the utilization of cocoa powder by gut microbes.

On the other hand, when the production of SCFA was studied, a similar tendency was observed: whereas the location of the crop did not have any influence, the roasting process changed the SCFA profile (Fig. 2B). Raw cocoa produced significantly higher concentrations of acetate and propionate whereas roasted cocoa fermentation resulted in significantly higher production of butyrate. All three SCFA are essential for the host's gut health. Among many other investigated properties, all three SCFA play a main role in maintaining the gut barrier, while butyrate is used as a substrate by epithelial cells and has also been linked to anti-inflammatory activity, having a major impact in keeping the gut healthy. ${ }^{46}$ As stated above, the chemical changes that originated during roasting could be the reason behind these differences in SCFA production. Although fiber is the main source for SCFA production, melanoidins have been demonstrated to be a source of SCFA when fermented by gut microbes. ${ }^{18}$ The higher production of butyrate in roasted samples suggests that some bacterial species that are able to produce butyrate could be growing better than in raw samples, or at least, that they can use non-enzymatic browning compounds (i.e. melanoidins) to generate a better yield of butyrate. In order to confirm whether the microbial community structure and SCFA production were correlated or associated, we performed a coinertia analysis. As it can be seen in Fig. 3C, there is a strong correlation ( $\mathrm{RV}=0.61 ; p=0.01$ ) between the SCFAbased PCA and the genus-based PCoA coordinations, since the distance between the same samples (depicted with an arrow) is usually less than that between different samples. This indicates that SCFA and gut microbial composition analyses supported each other.
According to the information presented so far, whether cocoa beans are roasted or not has a deep and significant impact on both the gut microbiota composition and its ability to produce SCFA. Therefore, the next step would be to unravel the most discriminant genera between the microbial communities resulting from raw and roasted cocoa fermentations (i.e. the genera that best describe or represent raw or roasted samples). A random forest was performed as discriminant analysis, since it is widely used to find features (in this case bacterial genera) to distinguish between different microbial communities. ${ }^{47}$ The most important genera to classify the samples, in this case as raw or roasted, are given by the variables Mean Decrease Accuracy and Mean Decrease Gini (see Material and methods section) and the higher they are the more influence they have in the classification. As depicted in Fig. 4A and B, among the top five positions for both variables can be found Veillonella, Faecalibacterium and Lachnospiraceae UCG 004. Whereas Veillonella is a propionate producer, Faecalibacterium is a butyrate producer and it also plays a positive role in the regulation of the immune system. ${ }^{43}$ Veillonella was found in much higher abundance in raw samples (Fig. 3C), which could explain in part the higher propionate concentrations in those samples. It is also especially significant to have found Faecalibacterium as a top discriminant bacterium, since it is a well-known butyrate producer and therefore it could explain, at least in part, the higher butyrate production with roasted samples. In addition, this could mean that Faecalibacterium can use non-enzymatic browning products (such as melanoidins) as fermentation substrates. This result would be in line with previous research showing that Faecalibacterium was favored by melanoidin fermentation. ${ }^{18}$ On the other hand, Roseburia and Butyricicoccus, both of them butyrate producers, ${ }^{43}$ were also found at higher abundance in roasted samples. Fig. 4C shows all those differentially $(p<$ 0.05) abundant genera between raw and roasted samples, some of them having been proven to have beneficial health effects or to produce SCFA. ${ }^{43,46}$

Results have clearly shown how the gut microbial fermentation of roasted and raw cocoa results in fairly different microbial communities and SCFA production. However, it is also important to know whether the degree of roasting generates significant differences. We studied the effect of the roasting degree in the top 7 discriminating genera (i.e. those genera with Mean Decrease Accuracy above 4 and Mean Decrease Gini above 0.3). As depicted in Fig. 5A, the roasting degree (temperature applied) did not have an influence on these bacteria. However, as it has been described before, ${ }^{48}$ furanic compounds such as HMF and furfural could inhibit the growth of certain bacteria. Thus, the potential inhibitory effects were checked by searching for Spearman correlations between HMF and furfural and any of the genera observed in our microbial communities. Negative correlations were found between those compounds and Blautia and Veillonella (Fig. 5B), though these correlations were significant only in the case of HMF. Still, as these are only correlations, other interactions could be involved, such as inhibition by other compounds generated 
A

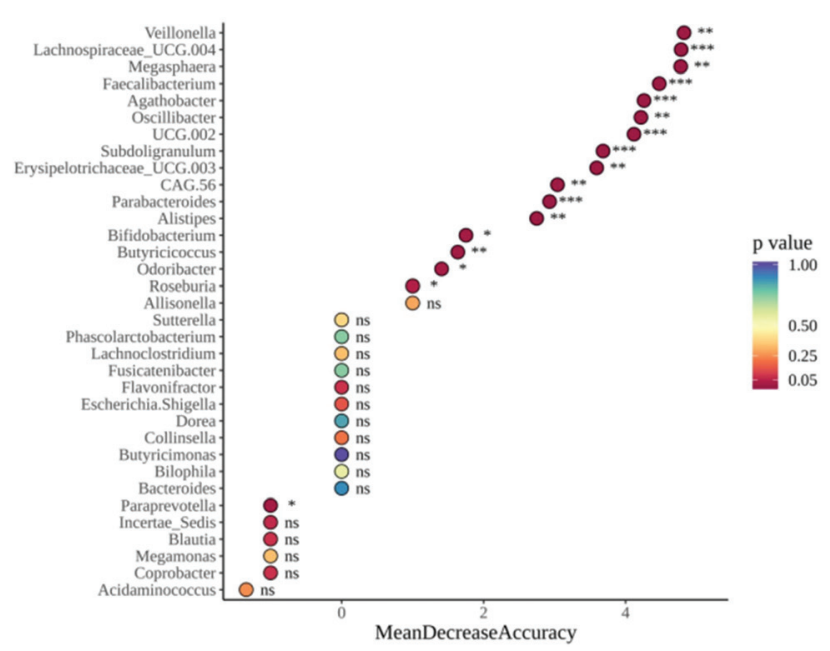

B

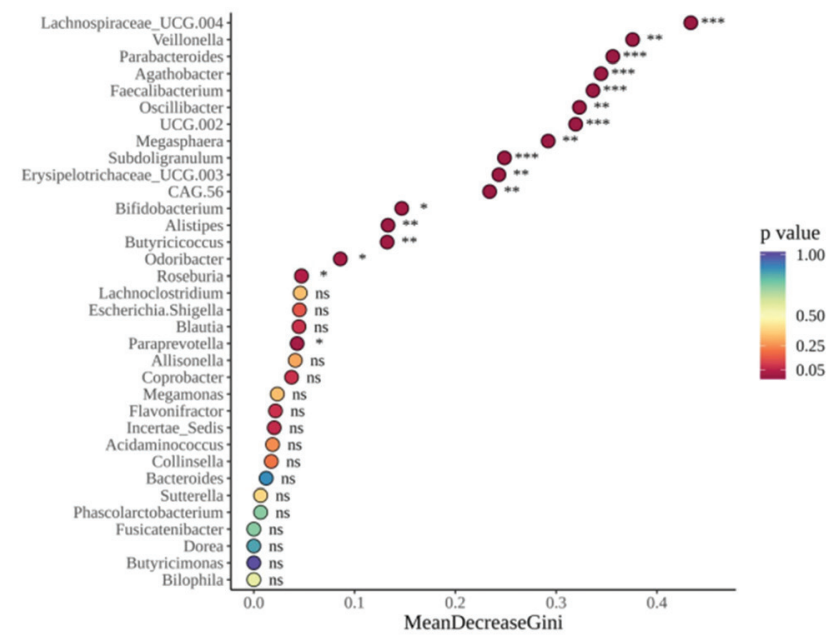

C $\square$ Raw $\square$ Roast

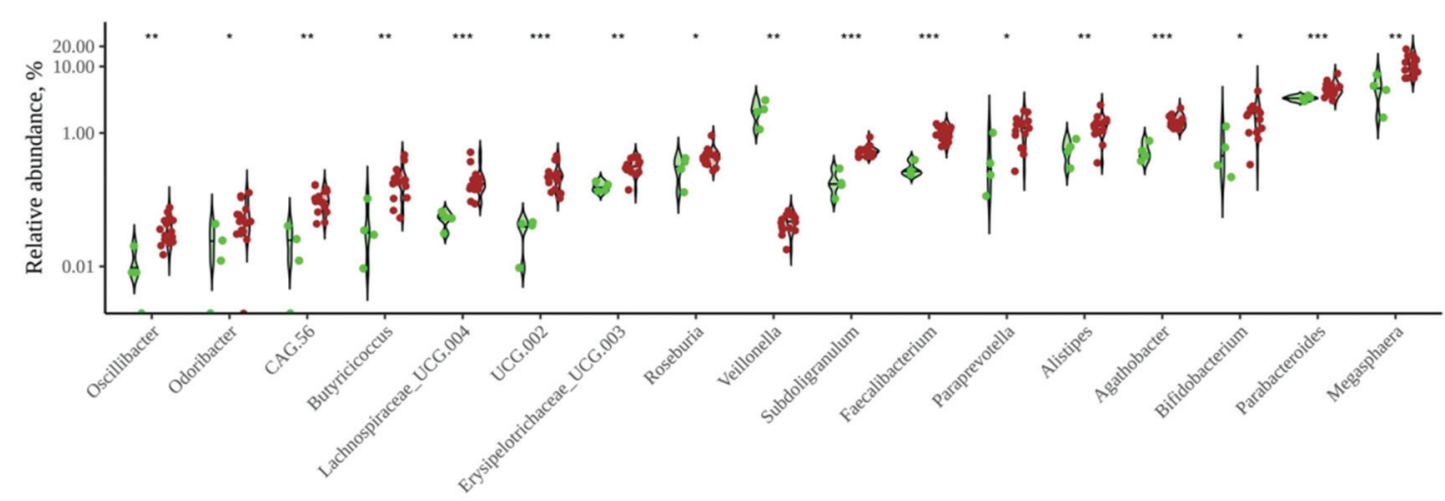

Fig. 4 (A) The mean decrease accuracy values for each genus included in random forest analysis. Mean decrease accuracy gives a rough estimate of the loss in prediction performance when that particular genus is omitted from the training set. (B) Mean decrease Gini values for each genus included in random forest analysis. Mean decrease Gini is a measure of node impurity; a node with the highest purity is that that only has features of the same class (roasted vs unroasted). By calculating the decrease in Gini when a feature is omitted, the importance of such feature for classification of the data as roasted or unroasted is highlighted. (C) Violin plot of all the differentially abundant genera between roasted and unroasted (raw) cocoa. Note the log scale (y axis). Significance labels: ns: not significant; *: $p<0.05 ; * *: p<0.01$; and ***: $p<0.001$. Statistical differences were calculated via Wilcoxon test using raw cocoa as the reference group.

during roasting or competition by other bacteria that are especially favored by other compounds generated during roasting. Conversely, a positive significant correlation between HMF and Alistipes was found. Similarly, this correlation is likely to be due to other compounds that appear during roasting and that can be used by bacteria (such as melanoidins) or to the fact that roasting causes the loss of compounds essential for other bacteria (such as poly- or oligosaccharides), allowing Alistipes to out-compete them and grow in their place. Finally, though acetate and propionate production seemed to decrease with temperature, differences between different roasting temperatures were not significant (Fig. 5C). Butyrate production was also affected differentially by temperature, reaching the highest concentrations at 50 and $70{ }^{\circ} \mathrm{C}$. However, again, differences were only significant between raw and roasted cocoa.

\subsection{Antioxidant capacity and thermal damage markers of chocolates}

Antioxidant capacity and thermal damage markers were also analyzed in the chocolates that contain the studied cocoa powder (provided by cocoa roasters). As shown in Table 1, the antioxidant capacity of the digested fraction was 2-3 times lower than that of the fermented fraction, except in the case of the Folic-Ciocalteu method, where similar results were found. In this sense, for the total phenolic compound analysis, the results obtained in this study were in line with those of other vegetable products ${ }^{26}$ and tailor-made chocolate products ${ }^{37}$ and 10-20 times higher than those reported by other authors for chocolate products. ${ }^{49}$ This could be related to the absence of the in vitro digestion-fermentation, which is known to release more antioxidant compounds than those obtained using solvent extraction. ${ }^{23}$ 
A
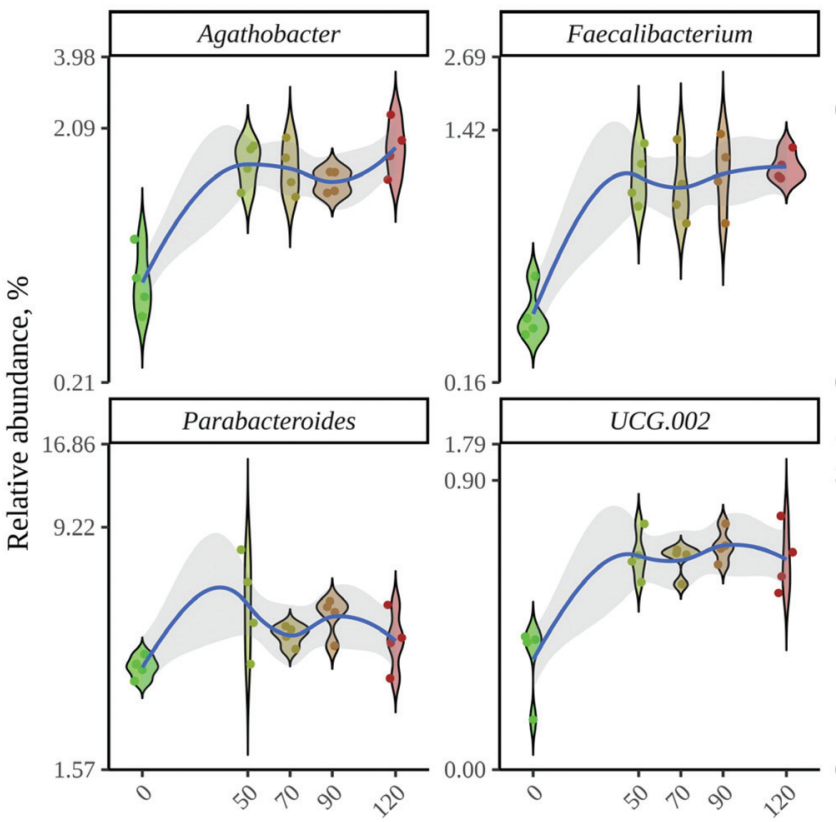

B
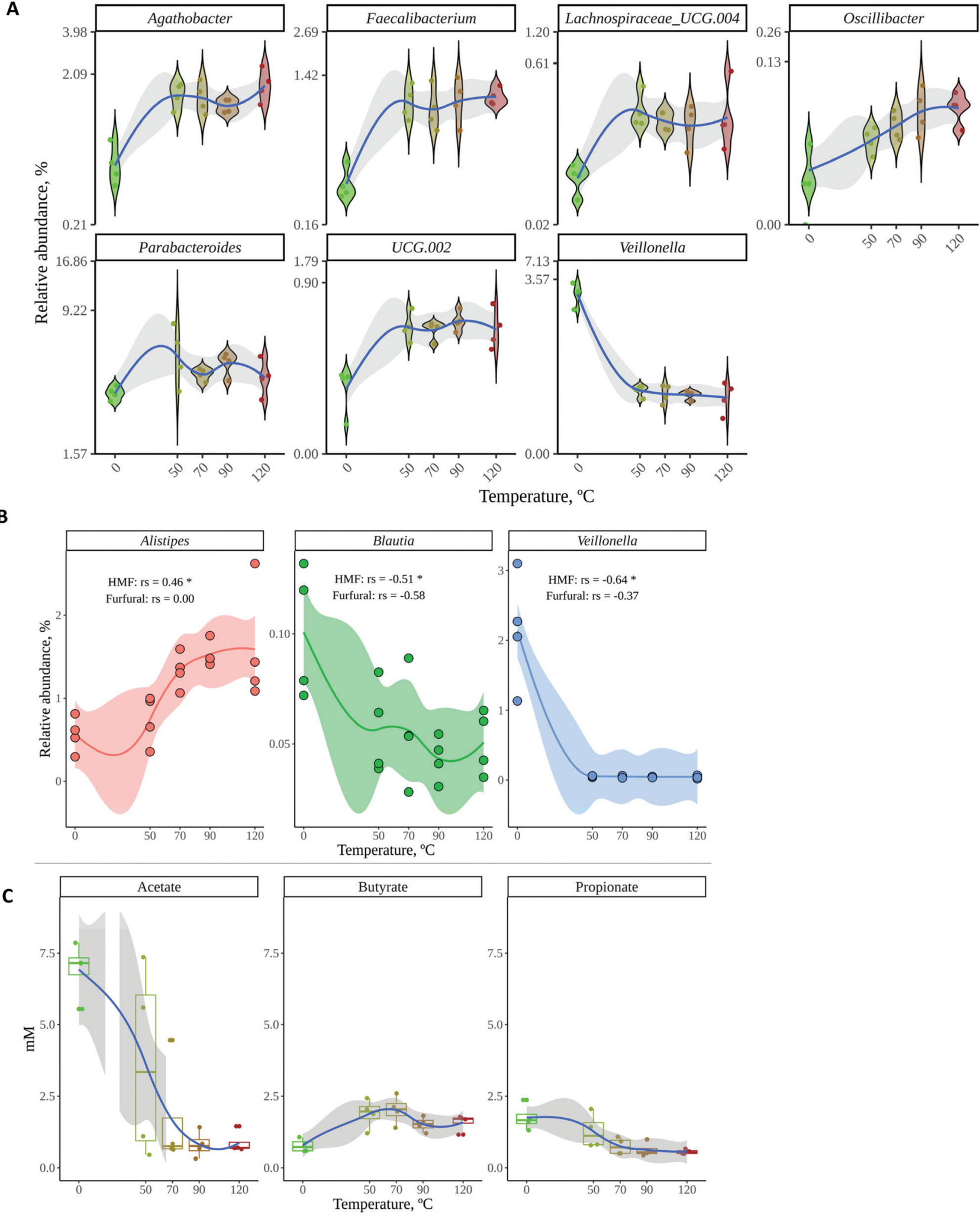

Fig. 5 (A) Relative abundance at each roasting temperature of the top 7 discriminant genera according to random forest. (B) Relative abundance at each roasting temperature of the genera that significantly correlated with either HMF or furfural concentration. Rs means Spearman correlation coefficient between relative genus abundance and metabolite concentration. Significance labels: ns: not significant; ${ }^{*} p<0.05$. (C) SCFA production at each roasting temperature. 
Table 1 Antioxidant capacity of chocolate products (different letters indicate significant differences $(p<0.05)$ between the digested and fermented groups)

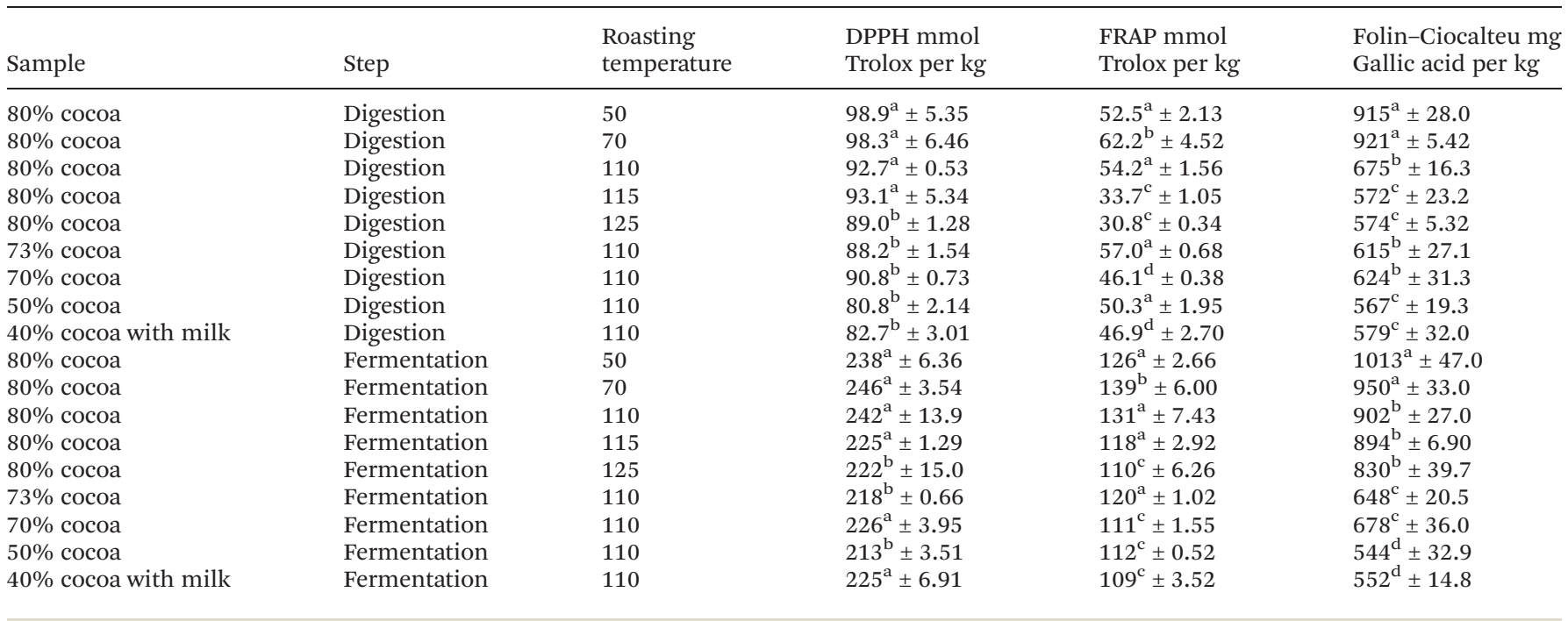

The antioxidant capacity followed the same tendency observed for cocoa powder: it decreases as the temperature applied is higher. The drop in antioxidant capacity becomes greater at 115 and $125{ }^{\circ} \mathrm{C}$, especially in the digested fraction. On the other hand, in the fermentation fraction, though the antioxidant capacity still decreases, the differences are weaker. A possible explanation could relate to the different compounds produced during microbial metabolism that may compensate, up to a certain extent, for the loss of natural antioxidants present in cocoa. These could be some newly generated compounds such as phenolic metabolites or compounds produced from melanoidin metabolism, but also other compounds that could be attached to polymers that reach the colon and are released once those polymers are degraded. In either case, during microbial fermentation neo-formed compounds are likely to appear, which not only makes the antioxidant capacity of this fraction higher than that of the digestion fraction (Table 1) but also softens the loss of antioxidant capacity due to roasting. ${ }^{50,51}$ In addition, as expected, the antioxidant capacity was higher in those chocolates with a higher cocoa percentage.

Finally, as with cocoa powder, HMF and furfural increased during roasting (Table 2) and a higher temperature resulted in higher concentrations of these compounds. The largest

Table 2 HMF and furfural contents of chocolate products (different letters indicate significant differences $(p<0.05)$ between different temperatures)

\begin{tabular}{llll}
\hline Sample & $\begin{array}{l}\text { Roasting } \\
\text { temperature }\end{array}$ & HMF mg kg $^{-1}$ & Furfural mg kg \\
\hline $80 \%$ cocoa & 50 & $0.437^{\mathrm{a}} \pm 0.464$ & $0.120^{\mathrm{a}} \pm 0.035$ \\
$80 \%$ cocoa & 70 & $1.144^{\mathrm{a}} \pm 0.171$ & $0.217^{\mathrm{b}} \pm 0.025$ \\
$80 \%$ cocoa & 110 & $3.258^{\mathrm{a}} \pm 0.286$ & $0.828^{\mathrm{a}} \pm 0.085$ \\
$80 \%$ cocoa & 125 & $3.125^{\mathrm{a}} \pm 0.183$ & $0.956^{\mathrm{a}} \pm 0.141$ \\
$40 \%$ cocoa with milk & 110 & $3.707^{\mathrm{b}} \pm 0.116$ & $1.101^{\mathrm{c}} \pm 0.162$
\end{tabular}

increase appeared from 70 to $110{ }^{\circ} \mathrm{C}$. Irrespective of the thermal treatment, the HMF levels of those chocolates included in our study were 10-30 times lower than those reported by Teixidó et $\mathrm{al}^{52}$ (from 42.1 to $74.4 \mathrm{mg} \mathrm{kg}^{-1}$ for dark and milk chocolates, respectively). The differences in HMF levels could be related to different thermal treatments and the cocoa percentage, since no information about these parameters was reported in that study.

\section{Conclusions}

This study describes the effect that different roasting temperatures, including no roasting, could have on the bioactivity of cocoa powder (antioxidant capacity and use by gut microbes) as well as the levels of HMF and furfural, as thermal damage markers. In the study, we analyzed cocoa samples from two different locations, but this factor did not show a significant influence on the experiments carried out. However, we did observe important differences depending on the roasting temperature. Whereas the antioxidant capacity decreased as the roasting temperature increased, HMF and furfural did the opposite. The loss of phenolic compounds during roasting has been stated to be the main cause for the drop in antioxidant capacity. On the other hand, during roasting, chemical browning takes place and HMF and furfural increase in concentration.

Gut microbial communities were significantly affected depending on whether cocoa was roasted or not regardless of the roasting temperature. Two of the most affected genera were Veillonella and Faecalibacterium. The former is a propionate producer and was found in significantly higher abundance in the raw cocoa samples, whereas the latter is a butyrate producer and was significantly more abundant in the roasted samples. This strengthens the findings regarding SCFA pro- 
duction, since it was found that the raw samples produced higher concentrations of acetate and propionate, while the roasted samples produced higher concentrations of butyrate. Additionally, Faecalibacterium has been associated with antiinflammatory effects and immune system modulation. Changes in gut microbial communities could be explained by the chemical changes that cocoa undergoes during roasting: loss of polyphenols, decrease in carbohydrates and chemical browning. In fact, it has been shown that melanoidins, the end products of non-enzymatic browning, have a prebiotic role. Therefore, consumption of roasted or unroasted cocoa could be preferred depending on the desired effect on the gut microbiota.

\section{Author contributions}

Conceptualization, S. P. C. and J. A. R. H.; methodology, L. Y. M. M., D. H. N., S. P. B., A. L.-A., S. R.-P., M. J. G., and M. P. F.; writing-original draft preparation, L. Y. M. M. and S. P. B.; writing-review and editing, S. P. B., M. P. F., S. P. C. and J. A. R. H.; funding acquisition, J. A. R. H. and S. P. B. All authors have read and agreed to the published version of the manuscript.

\section{Conflicts of interest}

The authors declare that there is no conflict of interest regarding the publication of this paper.

\section{Acknowledgements}

This work is part of the doctoral thesis of Lida Yaneth Maldonado-Mateus conducted within the context of the "Program of Nutrition and Food Sciences" at the University of Granada. This work was supported by the "Plan propio de Investigación y Transferencia" of the University of Granada under both programs "Intensificación de la Investigación, modalidad B" granted to José A. Rufián-Henares and the postdoctoral program "Perfeccionamiento de Doctores" in the case of Sergio Pérez-Burillo, and by the European Research Commission (Research Executive Agency) under the research project Stance4Health under a grant (Contract No. 816303) to José A. Rufián-Henares.

\section{References}

1 V. Voora, S. Bermúdez and C. Larrea, Global Market Report: Cocoa, 2019, p. 12.

2 I. Andújar, M. C. Recio, R. M. Giner and J. L. Ríos, Cocoa Polyphenols and Their Potential Benefits for Human Health, Oxid. Med. Cell. Longevity, 2012, 2012, 906252.

3 V. Sorrenti, S. Ali, L. Mancin, S. Davinelli, A. Paoli and G. Scapagnini, Cocoa Polyphenols and Gut Microbiota
Interplay: Bioavailability, Prebiotic Effect, and Impact on Human Health, Nutrients, 2020, 12, 1908.

4 D. Angelino, D. Carregosa, C. Domenech-Coca, M. Savi, I. Figueira, N. Brindani, S. Jang, S. Lakshman, A. Molokin, J. F. Urban, C. D. Davis, M. A. Brito, K. S. Kim, F. Brighenti, C. Curti, C. Bladé, J. M. del Bas, D. Stilli, G. I. SolanoAguilar and P. Mena, 5-(Hydroxyphenyl)- $\gamma$-ValerolactoneSulfate, a Key Microbial Metabolite of Flavan-3-ols, Is Able to Reach the Brain: Evidence from Different in Silico, In Vitro and In Vivo Experimental Models, Nutrients, 2019, 11, 2678.

5 W. J. Hollands, M. Philo, N. Perez-Moral, P. W. Needs, G. W. Savva and P. A. Kroon, Monomeric Flavanols Are More Efficient Substrates for Gut Microbiota Conversion to Hydroxyphenyl- $\boldsymbol{\gamma}$-Valerolactone Metabolites Than Oligomeric Procyanidins: A Randomized, PlaceboControlled Human Intervention Trial, Mol. Nutr. Food Res., 2000, 64, 1901135.

6 N. A. Febrianto and F. Zhu, Changes in the Composition of Methylxanthines, Polyphenols, and Volatiles and Sensory Profiles of Cocoa Beans from the Sul 1 Genotype Affected by Fermentation, J. Agric. Food Chem., 2020, 68, 8658-8675.

7 J. Delgado-Ospina, C. D. Di Mattia, A. Paparella, D. Mastrocola, M. Martuscelli and C. Chaves-Lopez, Effect of Fermentation, Drying and Roasting on Biogenic Amines and Other Biocompounds in Colombian Criollo Cocoa Beans and Shells, Foods, 2020, 9, 520.

8 M. S. Beg, S. Ahmad, K. Jan and K. Bashir, Status, supply chain and processing of cocoa-A review, Trends Food Sci. Technol., 2017, 66, 108-116.

9 J. A. Rufián-Henares, G. Guerra-Hernández and B. GarcíaVillanova, Colour measurement as indicator for controlling the manufacture and storage of enteral formulas, Food Control, 2006, 17, 489-493.

10 W. J. Hurst, S. H. Krake, S. C. Bergmeier, M. J. Payne, K. B. Mille and D. A. Stuart, Impact of fermentation, drying, roasting and Dutch processing on flavan-3-ol stereochemistry in cacao beans and cocoa ingredients, Chem. Cent. J., 2011, 5, 53.

11 F. Ioannone, C. D. Di Mattia, M. De Gregorio, M. Sergi, M. Serafini and G. Sacchetti, Flavanols, proanthocyanidins and antioxidant activity changes during cocoa (Theobroma cacao L.) roasting as affected by temperature and time of processing, Food Chem., 2015, 174, 256-262.

12 N. A. Febrianto and F. Zhu, Changes in the Composition of Methylxanthines, Polyphenols, and Volatiles and Sensory Profiles of Cocoa Beans from the Sul 1 Genotype Affected by Fermentation, J. Agric. Food Chem., 2020, 68, 8658-8675.

13 S. Molino, N. A. Casanova, J. A. Rufián-Henares and M. E. Fernández-Miyakawa, Natural Tannin Wood Extracts as a Potential Food Ingredient in the Food Industry, J. Agric. Food Chem., 2020, 68, 2836-2848.

14 J. A. Rufián-Henares, E. J. Guerra-Hernández and B. GarcíaVillanova, Maillard reaction in enteral formula processing: furosine, loss of o-phthaldialdehyde reactivity, and fluorescence, Food Res. Int., 2002, 35, 527-533. 
15 J. A. Rufián-Henares, B. García-Villanova and E. J. GuerraHernández, Generation of furosine and color in infant/ enteral formula-resembling systems, J. Agric. Food Chem., 2004, 52, 5354-5358.

16 M. A. Martín, S. Ramos, R. Mateos, J. A. Rufián-Henares, F. J. Morales, L. Bravo and L. Goya, Biscuit Melanoidins of Different Molecular Masses Protect Human HepG2 Cells against Oxidative Stress, J. Agric. Food Chem., 2009, 57, 7250-7258.

17 R. C. Borrelli and V. Fogliano, Bread crust melanoidins as potential prebiotic ingredients, Mol. Nutr. Food Res., 2005, 49, 673-678.

18 S. Pérez-Burillo, S. Rajakaruna, S. Pastoriza, O. Paliy and J. A. Rufián-Henares, Bioactivity of food melanoidins is mediated by gut microbiota, Food Chem., 2020, 316, 126309.

19 V. Fogliano, M. L. Corollaro, P. Vitaglione, A. Napolitano, R. Ferracane, F. Travaglia, M. Arlorio, A. Costabile, A. Klinder and G. Gibson, In vitro bioaccessibility and gut biotransformation of polyphenols present in the water-insoluble cocoa fraction, Mol. Nutr. Food Res., 2011, 55, S44S55.

20 M. Massot-Cladera, T. Pérez-Berezo, A. Franch, M. Castel and F. J. Pérez-Cano, Cocoa modulatory effect on rat faecal microbiota and colonic crosstalk, Arch. Biochem. Biophys., 2012, 527, 105-112.

21 D. Álvarez-Cilleros, S. Ramos, M. E. López-Oliva, F. Escrivá, C. Álvarez, E. Fernández-Millán and M. A. Martín, Cocoa diet modulates gut microbiota composition and improves intestinal health in Zucker diabetic rats, Food Res. Int., 2020, 132, 109058.

22 A. Brodkorb, L. Egger, M. Alminger, P. Alvito, R. Assunção, S. Ballance, T. Bohn, C. Bourlieu-Lacanal, R. Boutrou, F. Carrière, A. Clemente, M. Corredig, D. Dupont, C. Dufour, C. Edwards, M. Golding, S. Karakaya, B. Kirkhus, S. L. Feunteun and I. Recio, INFOGEST static in vitro simulation of gastrointestinal food digestion, Nat. Protoc., 2019, 14, 991-1014.

23 S. Pérez-Burillo, J. A. Rufián-Henares and S. Pastoriza, Towards an improved global antioxidant response method $($ GAR + ): Physiological-resembling in vitro digestion-fermentation method, Food Chem., 2018, 239, 1253-1262.

24 S. Pérez-Burillo, S. Molino, B. Navajas-Porras, A. J. ValverdeMoya, D. Hinojosa, A. López-Maldonado, S. Pastoriza and J. A. Rufián-Henares, An in vitro batch fermentation protocol to study the contribution of food on gut microbiota composition and functionality, Nat. Protoc., 2021, 16, 31863209.

25 I. F. Benzie and J. J. Strain, The ferric reducing ability of plasma (FRAP) as a measure of "antioxidant power": The FRAP assay, Anal. Biochem., 1996, 239, 70-76.

26 B. Navajas-Porras, S. Pérez-Burillo, A. J. Valverde-Moya, D. Hinojosa-Nogueira, S. Pastoriza and J. A. RufiánHenares, Effect of Cooking Methods on the Antioxidant Capacity of Plant Foods Submitted to In Vitro DigestionFermentation, Antioxidants, 2020, 9, 1312.
27 V. L. Singleton and J. A. Rossi, Colorimetry of Total Phenolics with Phosphomolybdic-Phosphotungstic Acid Reagents, Am. J. Enol. Vitic., 1965, 16, 144-158.

28 J. A. Rufián-Henares, B. García-Villanova and E. GuerraHernández, Determination of furfural compounds in enteral formula, J. Liq. Chromatogr. Relat. Technol., 2001, 24, 3049-3061.

29 A. Klindworth, E. Pruesse, T. Schweer, J. Peplies, C. Quast, M. Horn and F. O. Glöckner, Evaluation of General 16S Ribosomal RNA Gene PCR Primers for Classical and nextGeneration Sequencing- Based Diversity Studies, Nucleic Acids Res., 2013, 41, e1.

30 B. J. Callahan, P. J. McMurdie, M. J. Rosen, A. W. Han, A. J. A. Johnson and S. P. Holmes, DADA2: high-resolution sample inference from Illumina amplicon data, Nat. Methods, 2016, 13, 581-583.

31 B. Langmead and S. L. Salzberg, Fast gapped-read alignment with Bowtie 2, Nat. Methods, 2012, 9, 357-359.

32 C. Quast, E. Pruesse, P. Yilmaz, J. Gerken, T. Schweer, P. Yarza, et al., The SILVA ribosomal RNA gene database project: improved data processing and web-based tools, Nucleic Acids Res., 2013, 41, 590-596.

33 O. Paliy and V. Shankar, Application of multivariate statistical techniques in microbial ecology, Mol. Ecol., 2016, 25, 1032-1057.

34 A. Liaw and M. Wiener, Classification and Regression by random Forest, $R$ News, 2002, 2, 5.

35 S. Pérez-Burillo, J. A. Rufián-Henares and S. Pastoriza, Effect of home cooking on the antioxidant capacity of vegetables: Relationship with Maillard reaction indicators, Food Res. Int., 2019, 121, 514-523.

36 I. Rowland, G. Gibson, A. Heinken, K. Scott, J. Swann, I. Thiele and K. Tuohy, Gut microbiota functions: Metabolism of nutrients and other food components, Eur. J. Nutr., 2018, 57, 1-24.

37 B. Urbańska and J. Kowalska, Comparison of the Total Polyphenol Content and Antioxidant Activity of Chocolate Obtained from Roasted and Unroasted Cocoa Beans from Different Regions of the World, Antioxidants, 2019, 8, 283.

38 E. Fernández-Romero, S. G. Chavez-Quintana, R. Siche, E. M. Castro-Alayo and F. P. Cardenas-Toro, The Kinetics of Total Phenolic Content and Monomeric Flavan-3-ols during the Roasting Process of Criollo Cocoa, Antioxidants, 2020, 9, 146.

39 S. Pérez-Burillo, S. Pastoriza, N. Jiménez-Hernández, G. D’Auria, M. P. Francino and J. A. Rufián-Henares, Effect of Food Thermal Processing on the Composition of the Gut Microbiota, J. Agric. Food Chem., 2018, 66, 11500-11509.

40 C. Delgado-Andrade, J. A. Rufián-Henares and F. J. Morales, Lysine availability is diminished in commercial fibre-enriched breakfast cereals, Food Chem., 2007, 100, 725-731.

41 G. Sacchetti, F. Ioannone, M. De Gregorio, C. Di Mattia, M. Serafini and D. Mastrocola, Non enzymatic browning during cocoa roasting as affected by processing time and temperature, J. Food Eng., 2016, 169, 44-52. 
42 S. Pastoriza, J. Álvarez, A. Végvári, J. Montilla-Gómez, O. Cruz-López, C. Delgado-Andrade and J. A. RufiánHenares, Relationship between HMF intake and SMF formation in vivo: An animal and human study, Mol. Nutr. Food Res., 2017, 61, 1600773.

43 H. J. Flint, S. H. Duncan, K. P. Scott and P. Louis, Links between diet, gut microbiota composition and gut metabolism, Proc. Nutr. Soc., 2015, 74, 13-22.

44 D. Gniechwitz, N. Reichardt, M. Blaut, H. Steinhart and M. Bunzel, Dietary Fiber from Coffee Beverage: Degradation by Human Fecal Microbiota, J. Agric. Food Chem., 2007, 55, 6989-6996.

45 A. A. Kolodziejczyk, D. Zheng and E. Elinav, Diet-microbiota interactions and personalized nutrition, Nat. Rev. Microbiol., 2019, 17, 742-753.

46 D. Ríos-Covián, P. Ruas-Madiedo, A. Margolles, M. Gueimonde, C. G. de los Reyes-Gavilán and N. Salazar, Intestinal Short Chain Fatty Acids and their Link with Diet and Human Health, Front. Microbiol., 2016, 7, 185.

47 Y. H. Zhou and P. Gallins, A Review and Tutorial of Machine Learning Methods for Microbiome Host Trait Prediction, Front. Genet., 2019, 10, 579.
48 F. Monlau, C. Sambusiti, A. Barakat, M. Quéméneur, E. Trably, J. P. Steyer and H. Carrère, Do furanic and phenolic compounds of lignocellulosic and algae biomass hydrolyzate inhibit anaerobic mixed cultures? A comprehensive review, Biotechnol. Adv., 2014, 32, 934951.

49 D. Dumbrava, L. A. Popescu, C. M. Soica, A. Nicolin, I. Cocan, M. Negrea, E. Alexa, D. Obistioiu, I. Radulov, S. Popescu, C. Watz, R. Ghiulai, A. Mioc, C. Szuhanek, C. Sinescu and C. Dehelan, Nutritional, Antioxidant, Antimicrobial, and Toxicological Profile of Two Innovative Types of Vegan, Sugar-Free Chocolate, Foods, 2020, 9, 1844.

50 S. Pérez-Burillo, T. Mehta, A. Esteban-Muñoz, S. Pastoriza, O. Paliy and J. Á. Rufián-Henares, Food Chem., 2019, 279, 252-259.

51 W. Chen, X. Zhu, Q. Lu, L. Zhang, X. Wang and R. Liu, Food Res. Int., 2020, 135, 109271.

52 E. Teixidó, O. Núñez, F. J. Santos and M. T. Galcerán, 5Hydroxymethylfurfural content in foodstuffs determined by mecellar electrokinetic chromatography, Food Chem., 2011, 126, 1902-1908. 